\title{
An evolution of the IR-Radio correlation?
}

\author{
R. J. Beswick* \\ Jodrell Bank Centre for Astrophysics, The University of Manchester \\ E-mail: Robert.Beswick@manchester.ac.uk
}

T. W. B. Muxlow

Jodrell Bank Centre for Astrophysics, The University of Manchester

E-mail: twbmejb.man.ac.uk

\section{H. Thrall}

Jodrell Bank Centre for Astrophysics, The University of Manchester E-mail: hthrallejb.man.ac.uk

\section{A. M. S. Richards}

Jodrell Bank Centre for Astrophysics, The University of Manchester

E-mail: amsrejb.man.ac.uk

\section{S. T. Garrington}

Jodrell Bank Centre for Astrophysics, The University of Manchester

E-mail: stg@jb.man.ac.uk

Using extremely deep ( $\mathrm{rms} \sim 3.3 \mu \mathrm{Jy} / \mathrm{bm}$ ) $1.4 \mathrm{GHz}$ sub-arcsecond resolution MERLIN+VLA radio observations of a $8.5 \times 8.5$ field centred upon the Hubble Deep Field North, in conjunction with Spitzer $24 \mu \mathrm{m}$ data we present an investigation of the radio-MIR correlation at very low flux densities. By stacking individual sources within these data we are able to extend the MIR-radio correlation to the extremely faint ( $\sim$ microJy and even sub-microJy) radio source population. Tentatively we demonstrate a small deviation from the correlation for the faintest MIR sources. We suggest that this small observed change in the gradient of the correlation is the result of a suppression of the MIR emission in faint star-forming galaxies. This deviation potentially has significant implications for using either the MIR or non-thermal radio emission as a star-formation tracer at low luminosities.

From planets to dark energy: the modern radio universe

October 1-5 2007

University of Manchester, Manchester, UK

\footnotetext{
* Speaker.
} 


\section{Introduction}

Radio and infrared emission from galaxies in both the nearby and distant Universe is thought to arise from processes related to star-formation, hence resulting in the correlation between these two observing bands. The infrared emission is produced from dust heated by photons from young stars and the radio emission predominately arises from synchrotron radiation produced by the acceleration of charged particles from supernovae explosions. It has however recently been suggested that at low flux density and luminosities there may be some deviation from the tight well-known radio-IR correlation seen for brighter galaxies [1], 2].

[1] argue that while the IR emission from luminous galaxies will trace the majority of the star-formation in these sources, in low luminosity galaxies the IR emission will be less luminous than expected considering the rate of star-formation within the source (i.e. the IR emission will not fully trace the star-formation). In this scenario the reduced efficiency of IR production relative to the source star-formation rate (SFR) would be the result of inherently lower dust opacities in lower luminosity sources and consequently less efficient reprocessing of UV photons from hot young stars into IR emission. The simple consequence of this is that at lower luminosities the near linear

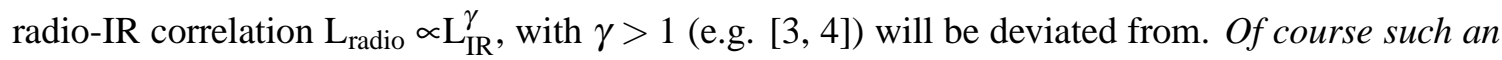
assertion is dependent upon the radio emission providing a reliable tracer of star-formation at low luminosities which may be equally invalid.

Recently [2] have presented a statistical analysis of Australia Telescope Compact Array (ATCA) $20 \mathrm{~cm}$ observations of the $24 \mu \mathrm{m}$ sources within the Spitzer Wide Field Survey (SWIRE). In this work [2] have co-added sensitive ( $\mathrm{rms} \sim 30 \mu \mathrm{Jy}$ ) radio data at the locations of several thousand $24 \mu \mathrm{m}$ sources. Using this method they have statistically detected the microJy radio counterparts of faint $24 \mu \mathrm{m}$ sources. At low flux densities $\left(\mathrm{S}_{24 \mu \mathrm{m}}=100 \mu \mathrm{Jy}\right)$ they confirm the IR-radio correlation but find it to have a lower coefficient $\left(\mathrm{S}_{1.4 \mathrm{GHz}}=0.039 \mathrm{~S}_{24 \mu \mathrm{m}}\right)$ than had previously been reported at higher flux densities. This coefficient is significantly different from results previously derived from detections of individual objects (e.g. [5]) and is speculated by [2] to be the result of a change in the slope of the radio-IR correlation at low flux densities.

In this work (which is described in more detail in [6, 7) we have utilised very deep, high resolution $20 \mathrm{~cm}$ observations of the Hubble Deep Field North and surrounding area made using MERLIN and the VLA [8] in combination with publicly available $24 \mu \mathrm{m}$ Spitzer source catalogues from GOODS to study the MIR-Radio correlation for microjansky radio sources. This study extends the flux density limits of the radio-IR correlation by more than an order of magnitude for individual sources and overlaps the flux density regime studied using statistical stacking methods by other authors.

\section{Observations}

\subsection{Radio Data}

Extremely deep radio observations of the HDF-N region were made in 1996-97 at $1.4 \mathrm{GHz}$ using both MERLIN and the VLA. These observations were initially presented in [8], [9] and [10]. The results from the combined 18 day MERLIN and $42 \mathrm{hr}$ VLA observations are described in detail 

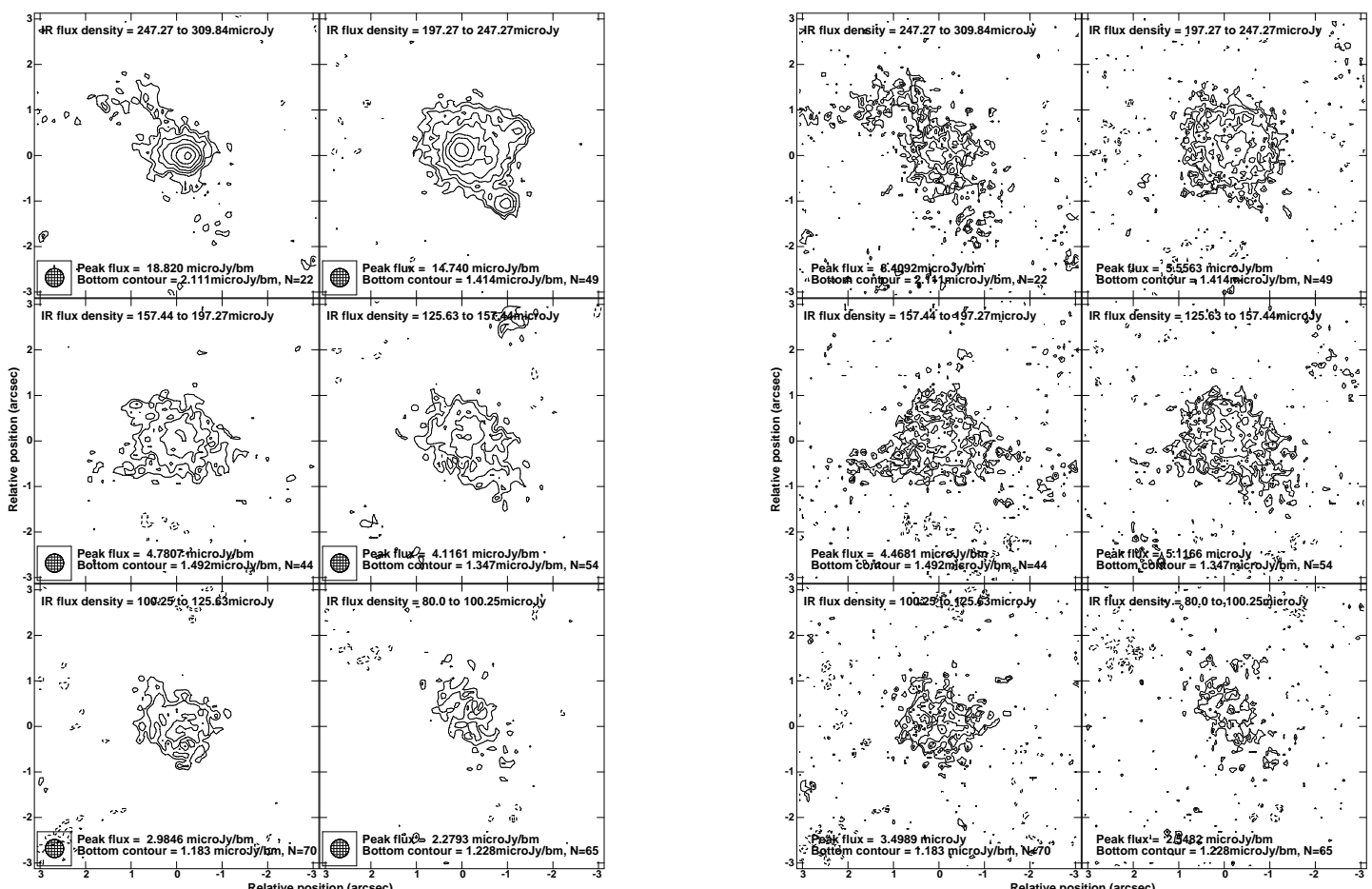

Figure 1: Mean (left) and median (right) images of the $1.4 \mathrm{GHz}$ radio emission for all sources within the six faintest $24 \mu$ m flux density logarithmic bins plotted in Fig. 1 in descending flux density order from top-left to bottom-right. The range of $24 \mu \mathrm{m}$ flux density over which each image has been stacked is shown at the top of individual panels. Each image is contoured with levels of $-2,-1.414,-1,1,1.414,2,2.828,4,5.657,8$, $11.31,16,22.63$ and 32 times $3 \times(3.3 / \sqrt{\mathrm{N}}) \mu \mathrm{Jy} \mathrm{bm}^{-1}$, where $\mathrm{N}$ equals the number of $24 \mu \mathrm{m}$ source positions averaged in the map. The peak flux density, lowest plotted contour and number of IR sources which have been averaged over $(\mathrm{N})$ is listed at the bottom of each image panel.

in [8]. The combined MERLIN+VLA image has an rms noise level of $3.3 \mu \mathrm{Jy}$ per 0. "2 circular beam making it amongst the most sensitive, high-resolution radio images made to date.

\subsection{GOODS-N Spitzer $24 \mu \mathrm{m}$ observations}

As part of the GOODS enhanced data release ${ }^{1}$ (DR1+ February 2005) a catalogue of Spitzer $24 \mu \mathrm{m}$ source positions and flux densities for the GOODS-N field were released. This source catalogue is limited to flux densities $>80 \mu$ Jy providing a highly complete and reliable sample. At the time of writing this $24 \mu \mathrm{m}$ source catalogue represents the most complete and accurate midinfrared source list publicly available for the GOODS-N/HDF-N field.

All $24 \mu \mathrm{m}$ sources which are detected optically in GOODS HST ACS images show an accurate astrometric alignment with their optical counterparts implying that the astrometry between these two data-sets and their subsequent catalogues is self-consistent. However, a comparison of the astrometric alignment of the positions of sources catalogued by GOODS derived from their HST ACS images [11, 7] shows there to be a systematic offset in declination of -0 "' 342 from the radio reference frame. This linear declination correction, although small relative to the Spitzer resolution

\footnotetext{
${ }^{1}$ http://www.stsci.edu/science/goods/DataProducts/
} 
at $24 \mu \mathrm{m}$ is significant when compared to these high resolution radio data. This linear correction has been applied to the Spitzer source positions prior to all comparisons between the two data sets.
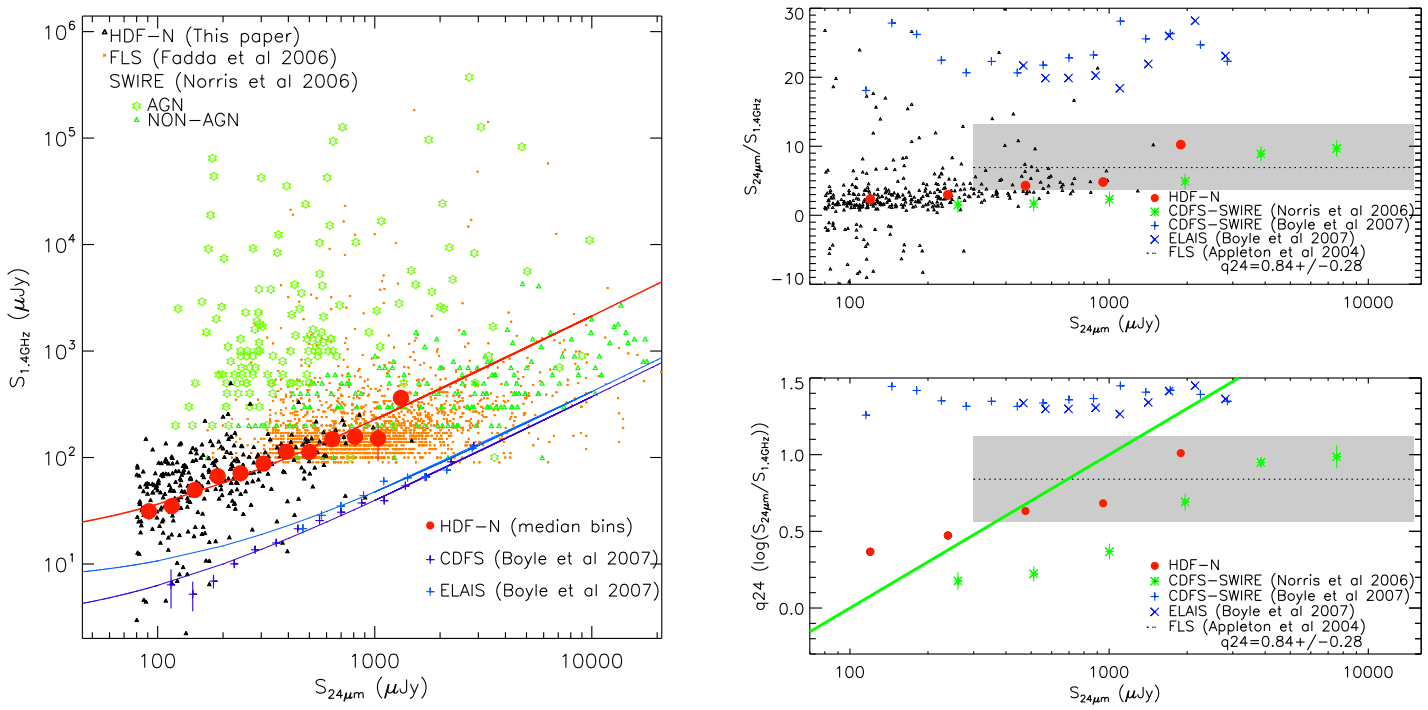

Figure 2: Left: Radio $1.4 \mathrm{GHz}$ versus the $24 \mu \mathrm{m}$ flux density. Sources from the $8.5 \times 8.5 \mathrm{HDF}-\mathrm{N}$ field are plotted individually (small black triangles). The median radio flux density logarithmically binned by $24 \mu \mathrm{m}$ flux densities are plotted as large filled red circles. The solid red line represents the best-fit line to the binned HDF-N data alone. Sources within the CDFS-SWIRE field detected at both $24 \mu \mathrm{m}$ and $1.4 \mathrm{GHz}$ from Norris et al. [12] are plotted as either green open stars (identified as AGN) or green triangles (not identified as AGN). All sources detected at both $1.4 \mathrm{GHz}$ and $24 \mu \mathrm{m}$ in the Spitzer First Look Survey (FLS) with source position separations of $<1$ !" 5 are plotted in orange [13]. Note the quantisation of the SWIRE and FLS points, in this and subsequent plots is a result of the accuracy of the flux densities tabulated in the literature. The blue pluses and fitted lines in the low portion of the plot show the IR-radio correlation derived from stacking radio emission at the positions of $24 \mu \mathrm{m}$ sources in the CDFS and ELAIS field by Boyle et al. [2]].

Right: In the upper panel the flux density ratio $\left(\mathrm{S}_{24} \mu \mathrm{m} / \mathrm{S}_{1.4 \mathrm{GHz}}\right)$ versus $24 \mu \mathrm{m}$ flux density is shown. The individual sources from the HDF-N field are plotted as small black triangles, the median values of these HDF-N source binned as a function of $S_{24 \mu m}$ as filled circles, green stars show the median binned values for sources listed as non-AGN within the CDFS-SWIRE sample of Norris et al. [12] and the blue 'crosses' and 'pluses' show the flux density ratios derived from the stacking analysis of the SWIRE-CDFS and ELAIS fields respectively from Boyle et al. [2]. The overlaid black dotted line is the mean value for $\left(\mathrm{S}_{24 \mu \mathrm{m}} / \mathrm{S}_{1.4 \mathrm{GHz}}\right)$ derived by Appleton et al. [5]. This line is equivalent to $\mathrm{q}_{24}=0.84 \pm 0.28$ with the gray filled box representing the area enclosed by these errors and the flux density range investigated by Appleton et al.. The values of $\mathrm{q}_{24}$ against $24 \mu \mathrm{m}$ flux density are plotted in the lower panel. The symbols within this plot are identical to those in the upper panel, individual HDF-N sources are not included for clarity. The additional diagonal solid green line represents a line of constant radio flux density of $100 \mu \mathrm{Jy}$, the lowest flux density of sources in the SWIRE sample plotted here (sources above this line are excluded by this limit from the SWIRE sample). This flux density limit will significantly effect the values of the binned SWIRE data points (green crosses) negatively biasing the four lowest flux density bins. This bias only effects the SWIRE sample. 


\section{Results \& Discussion}

Using these two highly sensitive data sets the radio emission from a sample of faint $24 \mu \mathrm{m}$ galaxies has been invesitigated. Of the 377 Spitzer sources within the radio field 303 were found to have total radio flux denisties in excess of 3 times the local rms in our deep radio imaging. Many of these sources, however, have peak flux densities comparable or below the point source detection threshold of these radio data.

By using both statistically stacked images and radio flux density measurements at the positions of individual Spitzer $24 \mu \mathrm{m}$ sources we have investigated the radio structures and flux densities of faint IR sources. In figure 1 the statistically averaged radio emission from faint $24 \mu \mathrm{m}$ galaxies in the GOODS-N field are shown. Each of these images is the statistically combined radio emission from the location of several tens of Spitzer $24 \mu \mathrm{m}$ sources and has been contoured at multiples of three times $3.3 \mu \mathrm{Jy}_{\text {beam }}{ }^{-1}$ divided by $\sqrt{\mathrm{N}}$ where $\mathrm{N}$ equals the number of Spitzer source positions that have been stacked together. As can be seen in these images the off-source noise levels achieved approaches the value expected when co-adding multiple images with near-Gaussian noise properties. The co-added image rms achieved in the faintest $24 \mu \mathrm{m}$ flux density bin (80.0 to $100.25 \mu \mathrm{Jy}$ ) is 0.45 and $0.56 \mu \mathrm{Jy}_{\text {beam }}{ }^{-1}$ in the mean and median co-added images respectively.

The Gaussian fitted sizes of the radio emission in the stacked images (Fig 1 ) provide an upper limit on the average size of the radio counterparts of these faint IR sources. The largest angular sizes of the radio emission in the median stacked images created from this sample range between 1 ". 4 and $2^{\prime \prime}$. This is approximately equivalent to a linear size of $10 \mathrm{kpc}$ at redshifts beyond 1 . These upper limits on the radio source sizes are consistent with radio emission on galactic and sub-galactic scales and originating within kpc-scale starburst systems.

By combining the Spitzer $24 \mu \mathrm{m}$ flux densities and the extracted $1.4 \mathrm{GHz}$ radio flux densities for these sources it is possible to begin to characterise the radio emission of the faintest IR galaxies. Shown in the left-hand portion of Fig. 2 is $1.4 \mathrm{GHz}$ total flux density of sources within the $8.5 \times 8.5$ MERLIN+VLA field plotted against their $24 \mu \mathrm{m}$ flux density. As a direct comparison also overlaid on this diagram are source flux densities from various other deep multi-wavelength observational campaigns of different regions of sky. As can be seen these new HDF-N/GOODS-N data significantly extend the observed IR-radio correlation for galaxies between these two wavelengths.

Binning and re-plotting these data in terms of $\mathrm{S}_{24 \mu \mathrm{m}} / \mathrm{S}_{1.4 \mathrm{GHz}}$ and $\mathrm{q}_{24}\left(\log \left(\mathrm{S}_{24 \mu \mathrm{m}} / \mathrm{S}_{1.4 \mathrm{GHz}}\right)\right.$ (see Fig. 2 2 right) clearly show the slope and tightness of this correlation. However, it can be seen from Fig. 2 that these data appear to show that this correlation begins to deviate for the faintest sources. This deviation is small (and tentative) but implies that the faintest galaxies are under-luminous at MIR wavelengths relative to their observed radio wavelengths. This deviate, whilst small, has potentially direct implications on the use of either of these bands to quantify star-formation rates in faint galaxies. A more complete discussion of these results and their implications can be found in [6, 7].

\section{Conclusion}

Using one of the deepest high-resolution $1.4 \mathrm{GHz}$ observations made to date, in conjunction with deep $24 \mu \mathrm{m}$ Spitzer source catalogues from GOODS, we have investigated the microJy radio 
counterparts of faint MIR sources. These observations confirm that the microJy radio source population follow the MIR-radio correlation and extend this correlation by several orders of magnitude to very low flux densities and luminosities, and out to moderate redshifts. This extension of the MIR-radio correlation confirms that the majority of these extremely faint radio and $24 \mu \mathrm{m}$ sources are predominantly powered by star-formation with little AGN contamination.

Statistically stacking the radio emission from many tens of faint $24 \mu \mathrm{m}$ sources has been used to characterise the size and nature of the radio emission from very faint IR galaxies well below the nominal radio sensitivity of these data. Using these methods the MIR-radio correlation has been further extended and a tentative deviation in this correlation at very low $24 \mu \mathrm{m}$ flux densities has been identified.

\section{References}

[1] Bell, Eric, F. 2003, ApJ, 586, 794

[2] Boyle, B. J., Cornwell, T. J., Middelberg, E., Norris, R. P., Appleton, P. N., Smail, Ian, 2007, MNRAS, 376, 1182

[3] Cox, M. J., Eales, S. A. E., Alexander, P., Fitt, A. J., 1988, MNRAS, 235, 1227

[4] Price, Rob., Duric, Nebojsa., 1992, ApJ, 401, 81

[5] Appleton, P. N., Fadda, D. T., Marleau, F. R., Frayer, D. T., Helou, G., Condon, J. J., Choi, P. I., Yan, L., Lacy, M., Wilson, G., Armus, L., Chapman, S. C., Fang, F., Heinrichson, I., Im, M., Jannuzi, B. T., Storrie-Lombardi, L. J., Shupe, D., Soifer, B. T., Squires, G. \& Teplitz, H. I., 2004 ApJS, 154, 147

[6] Beswick, R. J., Muxlow, T. W. B., Thrall, H., Richards, A. M. S., 2007, ASP 380, Page 265 "At the Edge of the Universe, Latest results from the deepest astronomical surveys", Eds: Afonso, Jose; Ferguson, Henry C.; Mobasher, Bahram; Norris, Ray (astro-ph/0612077)

[7] Beswick, R. J., Muxlow, T. W. B., Thrall, H., Richards, A. M. S., 2008, MNRAS, 385, 1143, (astro-ph/0801.1035)

[8] Muxlow, T. W. B., Richards, A. M. S., Garrington, S. T., Wilkinson, P. N., Anderson, B., Richards, E. A., Axon, D. J., Fomalont, E. B., Kellermann, K. I., Partridge, R. B., Windhorst, R. A. 2005 MNRAS, 358,1159

[9] Richards, E. A., Kellermann, K. I., Fomalont, E. B., Windhorst, R. A., Partridge, R. B., 1998, AJ, 116, 1039

[10] Richards, E. A., 2000, ApJ, 533, 611

[11] Richards, A. M. S., Muxlow, T. W. B., Beswick, R. J., Allen, M. G., Benson, K., Garrett, M. A., Garrington, S. T, Gonzalez-Solarez, E., Harrison, P. A., Kettenis, M., Laing, R. A., Richards, E. A., Thrall, H., van Langevelde, H. J., Walton, N. A., Wilkinson, P. N., Winstanley, N., 2007 A\&A 472, 805 (astro-ph/0706.3777)

[12] Norris, Ray P., Afonso, José, Appleton, Phil N., Boyle, Brian J., Ciliegi, Paolo, Croom, Scott M., Huynh, Minh T., Jackson, Carole A., Koekemoer, Anton M., Lonsdale, Carol J., Middelberg, Enno, Mobasher, Bahram, Oliver, Seb J., Polletta, Mari, Siana, Brian D., Smail, Ian, Voronkov, Maxim A., 2006, AJ, 132, 2409 
[13] Fadda, Dario, Marleau, Francine R., Storrie-Lombardi, Lisa J., Makovoz, David, Frayer, David T., Appleton, P. N., Armus, L., Chapman, S. C., Choi, P. I., Fang, F., Heinrichsen, I., Helou, G., Im, M., Lacy, M., Shupe, D. L., Soifer, B. T., Squires, G. K., Surace, J., Teplitz, H. I., Wilson, G., Yan, L., 2006, AJ, 131, 2859 